\title{
Clinical Manifestation of Hypercalcemia Caused by Adrenal Insufficiency in Hemodialysis Patients: A Case-series Study
}

\author{
Yukitoshi Sakao ${ }^{1}$, Takeshi Sugiura ${ }^{2}$, Takayuki Tsuji ${ }^{3}$, Naro Ohashi ${ }^{3}$, \\ Hideo Yasuda ${ }^{3}$, Yoshihide Fujigaki ${ }^{3}$ and Akihiko Kato ${ }^{1}$
}

\begin{abstract}
Objective The goal of this study was to clarify the clinical manifestation of hypercalcemia due to hypoadrenalism in hemodialysis (HD) patients.

Methods We retrospectively analyzed the clinical characteristics of five HD patients who had presented with hypercalcemia due to adrenal insufficiency (age: 69 \pm 7 [58-75] years old, time on HD: 13 \pm 11 [2-32] years). We conducted corticotropin-releasing hormone (CRH) and adrenocorticotropic hormone (ACTH) stimulation tests. We also examined serum bone turnover markers before and after glucocorticoid replacement.

Results All patients had critical illnesses at the onset of hypercalcemia. They had at least one symptom, such as eosinophilia, hypoglycemia, or fever. The prevalence of hypercalcemia due to adrenal insufficiency was $1.3 \%$ in maintenance HD patients on admission. The causes of adrenal insufficiency were isolated ACTH deficiency, pituitary apoplexy, pituitary atrophy, glucocorticoid withdrawal syndrome, and unilateral adrenalectomy. Serum calcium (Ca) levels corrected by serum albumin were maximally increased to 12.9 to $14.3 \mathrm{mg} / \mathrm{dL}$ in four anuric HD patients and mildly elevated to $10.4 \mathrm{mg} / \mathrm{dL}$ in a patient with residual diuresis. Their basal serum cortisol levels ranged from $<1.0$ to $15.4 \mu \mathrm{g} / \mathrm{dL}$. Single CRH injections failed to increase serum cortisol in any of the patients. Glucocorticoid replacement acutely normalized serum $\mathrm{Ca}$ and decreased levels of carboxy-terminal telopeptide of type I collagen, a marker of bone resorption.

Conclusion Adrenal insufficiency could therefore be an occult cause of hypercalcemia in anuric HD patients who are critically ill.
\end{abstract}

Key words: hypercalcemia, adrenal crisis, acute illness, bone resorption, anuria

(Intern Med 53: 1485-1490, 2014)

(DOI: 10.2169/internalmedicine.53.1104)

\section{Introduction}

Hypercalcemia is one of the most important electrolyte disorders, because it is often a clinical manifestation of unsuspected illness. In general, malignancy accounts for $70 \%$ cases of hypercalcemia, followed by primary hyperparathyroidism in $20 \%$, and other causes in $10 \%$ (e.g., granulomatous diseases [tuberculosis, sarcoidosis], drugs [vitamin D analogs, thiazide diuretic], thyrotoxicosis, milk-alkali syndrome, and immobilization) (1).

Adrenal insufficiency is rarely recognized as a latent cause of mild to moderate hypercalcemia (2). We previously reported a case of isolated adrenocorticotropic hormone (ACTH) deficiency presenting with hypercalcemia in a patient on long-term hemodialysis (HD) (3). However, little is known about the clinical manifestation of hypercalcemia due to adrenal insufficiency in the dialysis population, and this lack of knowledge could lead to missed diagnoses. This study was performed to clarify the clinical characteristics of hypercalcemia induced by adrenal insufficiency among HD patients.

\footnotetext{
${ }^{1}$ Blood Purification Unit, Hamamatsu University School of Medicine, Japan, ${ }^{2}$ Division of Nephrology, Seirei Mikatahara General Hospital, Japan and ${ }^{3}$ Internal Medicine I, Division of Nephrology, Hamamatsu University School of Medicine, Japan

Received for publication June 6, 2013; Accepted for publication February 9, 2014
}

Correspondence to Dr. Yukitoshi Sakao, yuksakao@hama-med.ac.jp 


\section{Materials and Methods}

We retrospectively examined the clinical characteristics according to the medical records of five HD patients who had presented with hypercalcemia due to adrenal insufficiency and had been treated in last two years. Adrenal insufficiency was readily diagnosed by standard corticotropinreleasing hormone $(\mathrm{CRH})$ and $\mathrm{ACTH}$ stimulation tests. Both tests were performed in the early morning under resting conditions after an overnight fast. The patients' serum cortisol and plasma ACTH levels were measured by electrochemiluminescence immunoassays.

After collecting basal blood samples, we intravenously injected human CRH $(100 \mu \mathrm{g})$ at bolus and then collected blood samples 15, 30, 60, 90, and 120 minutes after the injection. Normally, plasma ACTH concentration peaks in 15 to 30 minutes, while serum cortisol increases maximally at 30 to 60 minutes after the injection. We diagnosed patients as hypo-responsive if peak ACTH or cortisol levels were less than two-fold higher than their basal levels. ACTH stimulation tests were also performed using synthetic ACTH $(250 \mu \mathrm{g})$ injections. Blood samples were drawn at 0,30 , and 60 minutes after the injection. We diagnosed patients with adrenal insufficiency when their peak level was below 18-20 $\mu \mathrm{g} / \mathrm{dL}$.

We corrected the serum calcium (Ca) level with the following formula: corrected $\mathrm{Ca}=$ measured $\mathrm{Ca}+(4-$ serum albumin). Intact parathyroid hormone (PTH) (normal, 10-65 $\mathrm{pg} / \mathrm{mL}$ ) and 1,25(OH) $)_{2}$-vitamin $\mathrm{D}_{3}$ (normal, 20-60 pg/mL) were measured at the onset and resolution of hypercalcemia following glucocorticoid replacement. We also measured serum bone-specific alkaline phosphatase (BAP; normal range, male 3.7-20.9, female 3.8-22.6 $\mu \mathrm{g} / \mathrm{L}$ ) as a marker of bone formation, and carboxy-terminal telopeptide of type I collagen (ICTP, normal $<4.5 \mathrm{ng} / \mathrm{mL}$ ) or cross-linked Ntelopeptide of type I collagen (NTx; normal, male 9.5-17.7, female 10.7-24.0 nmol BCE/L) as markers of bone resorption.

\section{Results}

\section{Prevalence of hypercalcemia in admitted HD pa- tients}

We treated a total of $381 \mathrm{HD}$ patients admitted to our university hospital in the last two years (Fig. 1). We excluded 76 patients who had stopped HD treatment $(n=18)$ or had not undergone serum $\mathrm{Ca}$ measurements during admission $(\mathrm{n}=58)$. We analyzed serum $\mathrm{Ca}$ and clinical symptoms in the remaining $305 \mathrm{HD}$ patients. Twenty-nine (9.5\%) patients had adjusted serum Ca levels $>10.0 \mathrm{mg} / \mathrm{dL}$ on admission and had at least one hypoadrenalism-related clinical symptom, such as eosinophilia, hypoglycemia, or fever. When we excluded patients who had received vitamin D analogs and/or Ca-containing phosphate binders, there were

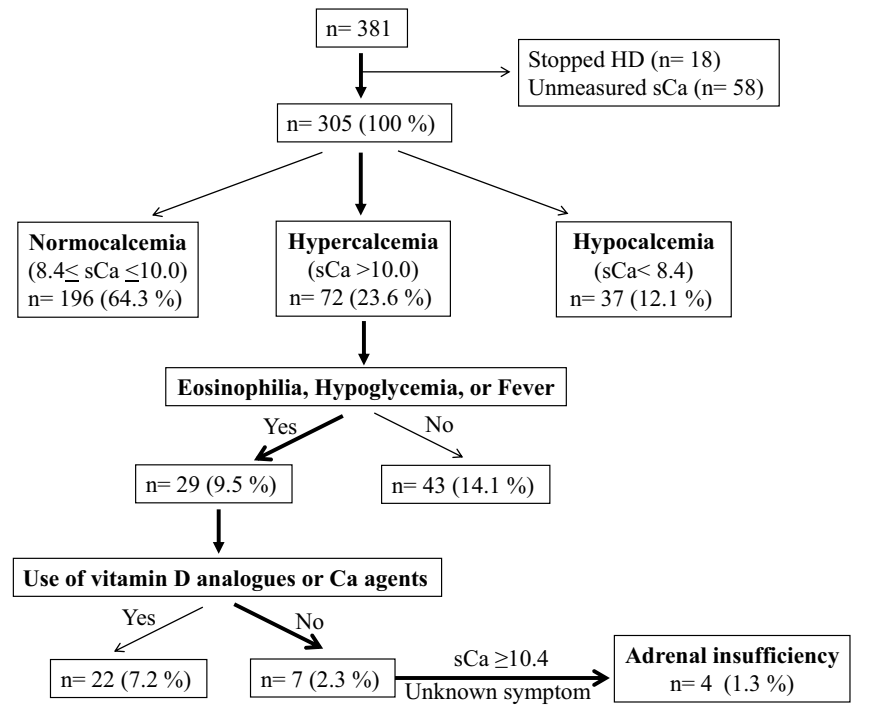

Figure 1. The prevalence of hypoadrenalism-induced hypercalcemia in HD patients on admission. Among the 305 maintenance HD patients, only four $\mathbf{( 1 . 3 \% )}$ ) were diagnosed as suffering from adrenal insufficiency-induced hypercalcemia (adjusted Ca $\geq 10.4 \mathrm{mg} / \mathrm{dL}$ ) in the tertiary hospital. HD: hemodialysis, sCa: corrected serum calcium, Ca: calcium

seven patients (2.3\%) who had concomitantly presented with hypercalcemia and clinical symptoms.

However, serum $\mathrm{Ca}$ had been mildly elevated (10.1 to $10.2 \mathrm{mg} / \mathrm{dL}$ ) in three patients, and their causes of fever were clinically evident. In addition, their Ca levels spontaneously normalized, so we did not test adrenal function in these patients. We measured cortisol and ACTH in the remaining four patients with overt hypercalcemia $(\mathrm{Ca} \geq 10.4 \mathrm{mg} / \mathrm{dL})$, and they were diagnosed as having adrenal insufficiency (cases 1-3, 5). Overall, the prevalence of hypoadrenalismrelated hypercalcemia in our hospital was $1.3 \%$ (four out of 305 patients). Case 4 had been diagnosed in another hospital (Seirei Mikatahara General Hospital).

\section{Clinical characteristics}

The clinical features of the five HD patients are listed in Table 1 . The patients' mean age was $69 \pm 7$ years old with a mean HD time of 13 years. The causes of end-stage kidney disease were primary chronic glomerulonephritis in three, diabetic nephropathy in one, and urolithiasis in one. Four patients were anuric (cases 1-4).

Before admission, active vitamin D analogs had been intravenously administered to all patients to control secondary hyperparathyroidism. However, these agents had been stopped soon after the development of overt hypercalcemia, and no patient had received any vitamin D analog at the assessment. There was also no patient who had taken calciumcontaining phosphate binders. The dialysate calcium concentrations were $2.5 \mathrm{mEq} / \mathrm{L}$ in cases 1 through 4 and 2.75 $\mathrm{mEq} / \mathrm{L}$ in case 5 . All patients had been undergoing regular HD three times per week for four hours each visit.

At the onset of hypercalcemia, all patients had been suf- 
Table 1. Clinical Characteristics in 5 Cases Presenting with Hypercalcemia

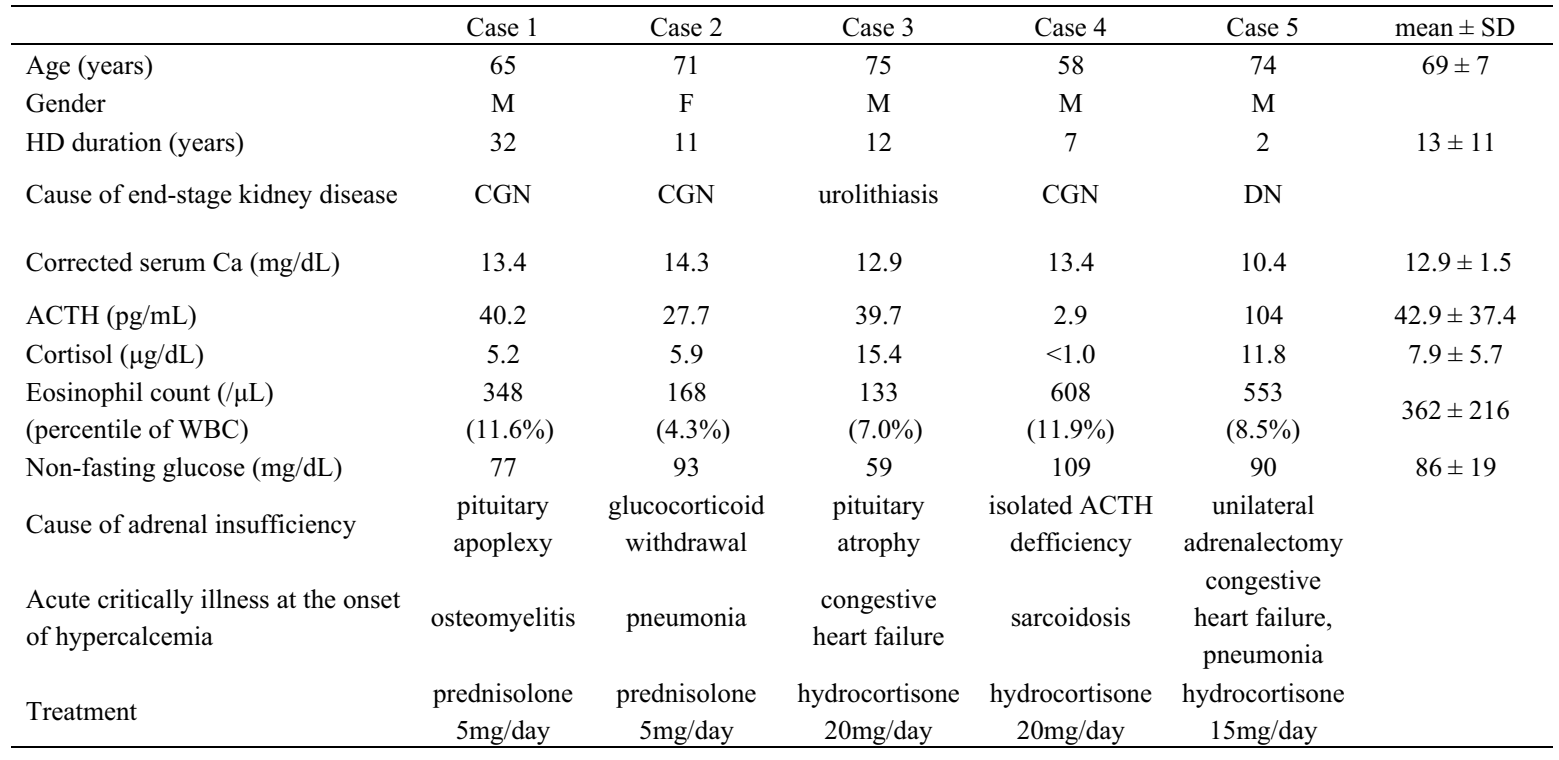

HD: hemodialysis, CGN: chronic glomerulonephritis, DN: diabetic nephropathy, Ca: calcium, ACTH: adrenocorticotropic hormone, WBC: white blood cell

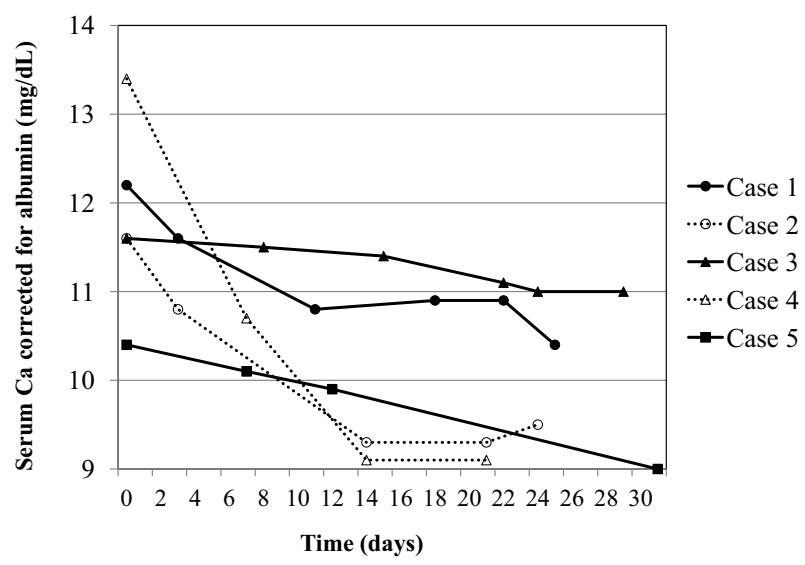

Figure 2. Changes in serum Ca after glucocorticoid replacement. Day 0 denotes the day on which prednisolone or hydrocortisone replacement was initiated. Glucocorticoid replacement decreased serum Ca. Ca: calcium

fering from a serious underlying illness. Cases 1 and 2 had been admitted to the intensive care unit (ICU) due to sepsis and severe pneumonia, respectively. Cases 3 and 5 had been complicated by acute respiratory failure due to congestive heart failure and pneumonia. Case 4 had a long history of persistent anorexia and general fatigue. Three patients (cases 1 through 3) had been suffering from persistent fever despite receiving systemic antibiotic therapy.

Laboratory examinations disclosed mild eosinophilia $\left(>500 / \mathrm{mm}^{3}\right.$ or $\geq 7 \%$ in differential leukocyte count) in four patients (except for case 2). Hypoglycemia was observed in two patients (cases 1 and 3). Serum cortisol levels ranged from $<1.0$ to $15.4 \mu \mathrm{g} / \mathrm{dL}$.

We presumed the patients as having imminent adrenal insufficiency in combination with clinical symptoms (nausea, wasting, lack of appetite, orthostatic hypotension, high fever with negative cultures, and unresponsive to antibiotic therapy), laboratory findings (hypoglycemia, eosinophilia), and random serum cortisol levels $<15 \mu \mathrm{g} / \mathrm{dL}$, which is a cut-off level to suspect hypoadrenalism in high-risk critically ill patients (4).

\section{CRH and ACTH stimulation tests}

In cases 1 through 4 , the peak levels of ACTH and cortisol were lower than two-fold their basal values (Table $2 \mathrm{~A}$ ). In case 5 , the increase in cortisol was not sufficient despite the normal ACTH response to exogenous CRH. There were two patients (cases 1 and 5) who had poorly responded to ACTH injection (Table 2B). We diagnosed case 5 as suffering from primary adrenal insufficiency due to unilateral adrenalectomy, and secondary adrenal sufficiency due to pituitary apoplexy, glucocorticoid withdrawal syndrome, pituitary atrophy, and isolated ACTH deficiency in cases 1 through 4 , respectively. No patient exhibited any abnormalities for other pituitary hormones.

\section{Effect of glucocorticoid treatment on $\mathrm{Ca}$ and bone metabolism}

The changes in serum $\mathrm{Ca}$ following glucocorticoid replacement are shown in Fig. 2 and Table 3. Serum Ca was substantially normalized within two to three weeks after replacement in four cases. In case 3, serum Ca gradually decreased during the first month but did not decline to within normal range $(\leq 10.0 \mathrm{mg} / \mathrm{dL})$. Because the patient had been suffering from secondary hyperparathyroidism, we administered cinacalcet hydrochloride $(25 \mathrm{mg} / \mathrm{day})$, and then serum Ca was substantially normalized.

Intact parathyroid hormone (PTH) was suppressed at the onset of hypercalcemia in all patients. Serum $1,25(\mathrm{OH})_{2}$ - 
Table 2. CRH and ACTH Stimulation Tests

\begin{tabular}{|c|c|c|c|c|c|c|c|}
\hline & & Basal & 15 & 30 & 60 & 90 & $120(\min )$ \\
\hline \multirow[t]{2}{*}{ Case 1} & ACTH & 36.9 & 55.4 & 40.6 & 31.4 & 24.9 & 16.6 \\
\hline & Cortisol & 5.6 & 6.4 & 7.2 & 7.2 & 6.6 & 6.3 \\
\hline \multirow[t]{2}{*}{ Case 2} & ACTH & 24.7 & 35.3 & 43 & 37.7 & 32.5 & 30.3 \\
\hline & Cortisol & 14 & 16.5 & 17.9 & 18.6 & 17 & 15.4 \\
\hline \multirow[t]{2}{*}{ Case 3} & АCTH & 69.7 & 87 & 90.9 & 83.3 & 63.5 & 69.6 \\
\hline & Cortisol & 13.6 & 14.3 & 15.9 & 15.1 & 14.5 & 14.3 \\
\hline \multirow[t]{2}{*}{ Case 4} & ACTH & 2.2 & NA & 3.8 & 3.5 & $<2.0$ & 2.3 \\
\hline & Cortisol & 1.9 & NA & 1.3 & 1.2 & 1.2 & 1.2 \\
\hline \multirow[t]{2}{*}{ Case 5} & ACTH & 112 & 245 & 222 & 192 & 154 & 115 \\
\hline & Cortisol & 14.2 & 17.4 & 18.5 & 17.9 & 17.2 & 17.2 \\
\hline
\end{tabular}

\begin{tabular}{cccc} 
B. ACTH stimulation test $(150 \mu \mathrm{g}$, iv) \\
\hline \multicolumn{5}{c}{ Basal } & 30 & $60(\mathrm{~min})$ \\
\hline Case 1 & 8.8 & 13.2 & 15.3 \\
Case 2 & 12.8 & 20.3 & 22.1 \\
Case 3 & 13.3 & 18.2 & 19.9 \\
Case 4 & NA & NA & NA \\
Case 5 & 12.9 & 17.2 & 18.6 \\
\hline NA. data not available & &
\end{tabular}

Table 3. Changes of Ca Metabolism and Bone Turnover Markers before and after Glucocorticoid Replacement

\begin{tabular}{|c|c|c|c|c|c|c|c|c|c|c|}
\hline & \multicolumn{2}{|c|}{ Case 1} & \multicolumn{2}{|c|}{ Case 2} & \multicolumn{2}{|c|}{ Case 3} & \multicolumn{2}{|c|}{ Case 4} & \multicolumn{2}{|c|}{ Case 5} \\
\hline & before & after & before & after & before & after & before & after & before & after \\
\hline Corrected Ca (mg/dL) & 12.0 & 10.9 & 11.2 & 9.5 & 12.5 & 11.2 & 13.4 & 9.1 & 10.4 & 9.9 \\
\hline Intact PTH (pg/mL) & 38.5 & 123.8 & 21.9 & 62.2 & 242.4 & 420.3 & 4.0 & 22.0 & 52.7 & 81 \\
\hline $1,25(\mathrm{OH})_{2}$-vitamin $\mathrm{D}_{3}(\mathrm{pg} / \mathrm{mL})$ & 16.2 & 19.2 & 14.7 & 6.8 & 10.7 & 16.1 & 34.5 & 3.3 & 17.8 & 12.3 \\
\hline ICTP $(\mathrm{ng} / \mathrm{mL})$ & 111.0 & 77.5 & 144.0 & 102.0 & 66.0 & 54.0 & NA & NA & 104 & 79 \\
\hline NTx (nmol BCE/L) & NA & NA & NA & NA & NA & NA & 114.0 & 67.0 & 37.8 & 31 \\
\hline BAP (IU/L) & 22.1 & 40.0 & 66.4 & 80.1 & 16.5 & 23.8 & 38.7 & 19.4 & 8.8 & 8.7 \\
\hline
\end{tabular}

vitamin $\mathrm{D}_{3}$ levels were also within normal ranges. PTHrelated protein levels were also in normal in all patients $(<1.1 \mathrm{pmol} / \mathrm{L})$. Glucocorticoid treatment restored intact PTH levels in all cases. Both the serum ICTP and NTx levels decreased after glucocorticoid replacement, while the serum BAP level was elevated in three patients.

\section{Discussion}

Adrenal insufficiency is known as an occult cause of mild to moderate hypercalcemia (5-8). In a series of 108 patients with idiopathic adrenal insufficiency, only $6(5.5 \%)$ patients were complicated by mild hypercalcemia (9). In a recent study, although $112(53 \%)$ of the 212 ICU patients were diagnosed as having latent adrenal insufficiency, no patients developed overt hypercalcemia (10). In this study, we found the prevalence of hypercalcemia due to adrenal insufficiency to be $1.3 \%$ in HD patients at a tertiary hospital.

It is presumed that increased Ca mobilization from bone tissue may be responsible for hypercalcemia due to adrenal insufficiency (11). Erosions in the phalanges of the hands on
X-ray film was observed in a patient with hypercalcemic crisis as presentation of Addison's disease (8). Fujikawa et al. (12) also found that urinary NTx was elevated in a hypercalcemic patient with post-partum thyrotoxicosis and adrenal insufficiency due to pituitary failure. A marked increase in urinary hydroxyproline, a marker of bone resorption, was described in a patient with hypercalcemia due to glucocorticoid withdrawal. Glucocorticoid therapy simultaneously normalized serum $\mathrm{Ca}$ and urinary excretion of hydroxyproline, suggesting that enhanced bone resorption could be responsible for hypercalcemia in patients with adrenal insufficiency (13).

ICTP and NTx increase in conjunction with impaired renal clearance (14). However, increased serum ICTP is positively histologically correlated with resorption severity in HD patients (15). Serum NTx is also correlated negatively with annual bone mineral density (BMD) change in the distal third of the radius in male patients on regular HD (16). Thus, both ICTP and NTx may be useful bone resorption markers in HD patients. In this study, glucocorticoid therapy decreased serum ICTP and NTx in HD patients experiencing 
adrenal crisis. We previously described a case of isolated ACTH deficiency in which serum $\mathrm{Ca}$ was normalized shortly after bisphosphonate administration without glucocorticoid replacement (3). Taken together, it is likely that increased bone resorption is at least, in part, associated with hypercalcemia in dialysis patients with adrenal insufficiency.

In this study, intact PTH was suppressed within a normal range in four HD patients, and serum $1,25(\mathrm{OH})_{2}$-vitamin $\mathrm{D}_{3}$ was decreased at the onset of hypercalcemia. An experimental study showed that serum PTH and $1,25(\mathrm{OH})_{2}$-vitamin $\mathrm{D}_{3}$ levels remain unchanged in dogs with hypoadrenocorticism and hypercalcemia (17). Elevated $\mathrm{Ca}$ was also reported in patients with hypoparathyroidism (18-20). Collectively, these observations suggest that adrenal failure increases serum $\mathrm{Ca}$ independent of the PTH-vitamin D axis.

Immobilization induces hypercalcemia $(21,22)$. Excess thyroid hormone stimulates bone resorption, which is partially inhibited by cortisol $(23,24)$. There have been some case reports of patients who concomitantly exhibited adrenal insufficiency and thyroid disorders, such as thyrotoxicosis $(12,25-27)$ and hypothyroidism $(28,29)$. However, in this study, there was no patient under immobilization or who had thyroid dysfunction before the onset of hypercalcemia.

Decreased glomerular filtration and increased tubular $\mathrm{Ca}$ reabsorption contribute to reduced Ca removal. In this study, anuric four patients had developed overt hypercalcemia ranging from 12.9 to $14.3 \mathrm{mg} / \mathrm{dL}$, which is similar to that described in a previous report (30). In contrast, one patient with preserved diuresis only presented with mild hypercalcemia $(10.4 \mathrm{mg} / \mathrm{dL})$. Therefore, impaired urine excretion of $\mathrm{Ca}$ could be an alternative mechanism in chronic HD patients.

Amyloid deposition is often observed in the adrenal and pituitary glands in dialysis patients. In an autopsy study of 15 HD patients, dialysis-related amyloid depositions were found in 26 out of 30 adrenal glands (31). Amyloid infiltration into the pituitary gland with adrenal failure has been reported in a HD patient with systemic amyloidosis (32). ACTH stimulation testing also revealed adrenal and pituitary defects in two out of five patients with end-stage kidney disease (33). In this study, three patients had been undergoing HD therapy for more than 10 years before the onset of hypercalcemia. It is reported that ACTH and cortisol responses to exogenous insulin-induced hypoglycemia was blunted in patients on long-term HD (34). As a result, long-term dialysis might predispose patients to the development of latent adrenal insufficiency via greater amyloid deposition in the adrenal and pituitary glands.

There are some limitations associated with this study. The sample size was too small to compare the mechanisms of hypercalcemia due to adrenal insufficiency and those due to other causes. We also could not exclude the influence of dialysis procedures on $\mathrm{Ca}$ metabolism in our patients. Because the initial levels of BAP were relatively low [mean 30.5 (8.8 to 66.4$) \mathrm{IU} / \mathrm{L}]$ in the present cases, their bone-buffering capacity for loaded $\mathrm{Ca}$ from dialysate may have been im- paired, which may be related to hypercalcemia.

In summary, our case-series study demonstrated that dialysis patients who presented with severe hypercalcemia due to adrenal insufficiency were anuric and had been undergoing long-term HD. Hypercalcemia became first evident during acute critical illnesses. Glucocorticoid replacement promptly normalized serum $\mathrm{Ca}$ and concomitantly reduced serum bone resorption marker levels. Therefore, adrenal insufficiency could be a rare latent cause of hypercalcemia in HD patients who are critically ill.

\section{The authors state that they have no Conflict of Interest (COI).}

\section{References}

1. Ziegler R. Hypercalcemic crisis. J Am Soc Nephrol 12: S3-S9, 2001.

2. Jacobs TP, Bilezikian JP. Rare causes of hypercalcemia. J Clin Endocrinol Metab 90: 6316-6322, 2005.

3. Kato A, Shinozaki S, Goga T, Hishida A. Isolated adrenocorticotropic hormone deficiency presenting with hypercalcemia in a patient on long-term hemodialysis. Am J Kidney Dis 42: E32-E36, 2003.

4. Cooper MS, Stewart PM. Corticosteroid insufficiency in acutely ill patients. N Engl J Med 348: 727-734, 2003.

5. Leeksma CH, De Graeff J, De Cock J. Hypercalcemia in adrenal insufficiency. Acta Med Scand 156: 455-458, 1957.

6. Pedersen KO. Hypercalcaemia in Addison's disease. Report on two cases and review of the literature. Acta Med Scand 181: 691698, 1967.

7. Siegler DI. Idiopathic Addison's disease presenting with hypercalcaemia. Br Med J 2: 522, 1970.

8. Downie WW, Gunn A, Paterson CR, Howie GF. Hypercalcaemic crisis as presentation of Addison's disease. Br Med J 1: 145-146, 1977.

9. Nerup J. Addison's disease-clinical studies: a report for 108 cases. Acta Endocrinol (Copenh) 76: 127-141, 1974.

10. Kromah F, Tyroch A, McLean S, Hughes H, Flavin N, Lee S. Relative adrenal insufficiency in the critical care setting: debunking the classic myth. World J Surg 35: 1818-1823, 2011.

11. Muls E, Bouillon R, Boelaert $J$, et al. Etiology of hypercalcemia in a patient with Addison's disease. Calcif Tissue Int 34: 523-526, 1982.

12. Fujikawa M, Kamihira K, Sato K, Okamura K, Kidota S, Lida M. Elevated bone resorption markers in a patient with hypercalcemia associated with post-partum thyrotoxicosis and hypoadrenocorticism due to pituitary failure. J Endocrinol Invest 27: 782-787, 2004.

13. Suzuki K, Nonaka K, Ichihara K, et al. Hypercalcemia in glucocorticoid withdrawal. Endocrinol Jpn 33: 203-209, 1986.

14. Ureña $P$, De Vernejoul MC. Circulating biochemical markers of bone remodeling in uremic patients. Kidney Int 55: 2141-2156, 1999.

15. Mazzaferro S, Pasquali M, Ballanti $P$, et al. Diagnostic value of serum peptides of collagen synthesis and degradation in dialysis renal osteodystrophy. Nephrol Dial Transplant 10: 52-58, 1995.

16. Maeno $Y$, Inaba M, Okuno $S$, Yamakawa $T$, Ishimura E, Nishizawa Y. Serum concentrations of cross-linked N-telopeptides of type I collagen: new marker for bone resorption in hemodialysis patients. Clin Chem 51: 2312-2317, 2005.

17. Gow AG, Gow DJ, Bell R, et al. Calcium metabolism in eight dogs with hypoadrenocorticism. J Small Anim Pract 50: 426-430, 2009 . 
18. Papadatos C, Klein R. Addison's disease in a boy with hypoparathyroidism. J Clin Endocrinol Metab 14: 653-660, 1954.

19. Farrell PM, Rikkers H, Moel D. Cortisol-dihydrotachysterol antagonism in a patient with hypoparathyroidism and adrenal insufficiency: apparent inhibition of bone resorption. J Clin Endocrinol Metab 42: 953-957, 1976.

20. Walker DA, Davies M. Addison's disease presenting as a hypercalcemic crisis in a patient with idiopathic hypoparathyroidism. Clin Endocrinol (Oxf) 14: 419-423, 1981.

21. Gopal H, Sklar AH, Sherrard DJ. Symptomatic hypercalcemia of immobilization in a patient with end-stage renal disease. Am J Kidney Dis 35: 969-972, 2000.

22. de Beus E, Boer WH. Denosumab for treatment of immobilization-related hypercalcemia in a patient with advanced renal failure. Clin Kidney J 5: 566-571, 2012.

23. Mundy GR, Shapiro JL, Bandelin JG, Canalis EM, Raisz LG. Direct stimulation of bone resorption by thyroid hormones. J Clin Invest 58: 529-534, 1976.

24. Jowsey J, Simons WS. Normocalcaemia in relation to cortisone secretion. Nature 217: 1277-1279, 1968.

25. Grossmann M, Fuller P, Hunter A, Teede H. Isolated ACTH deficiency presenting as severe heypercalcaemia. Clin Endocrinol (Oxf) 66: 603-604, 2007.

26. Vasikaran SD, Tallis GA, Braund WJ. Secondary hypoadrenalism presenting with hypercalcemia. Clin Endocrinol (Oxf) 41: 261264, 1994.
27. Wada $S$, Kurihara S, Imamaki K, et al. Hypercalcemia accompanied by hypothalamic hypopituitarism, central diabetes inspidus and hyperthyroidism. Intern Med 38: 486-490, 1999.

28. Miell J, Wassif W, McGregor A, Butler J, Ross R. Life-threatening hypercalcaemia in association with Addisonian crisis. Postgrad Med J 67: 770-772, 1991.

29. Katahira M, Yamada T, Kawai M. A case of Cushing syndrome with both secondary hypothyroidism and hypercalcemia due to postoperative adrenal insufficiency. Endocrine J 51: 105-113, 2004.

30. Montoli A, Colussi G, Minetti L. Hypercalcaemia in Addison's disease: calciotropic hormone profile and bone histology. J Intern Med 232: 535-540, 1992.

31. Barton CH, Vaziri ND, Gordon S, Eltorai I. Endocrine pathology in spinal cord injured patients on maintenance dialysis. Paraplegia 22: 7-16, 1984

32. Ozdemir D, Dagdelen S, Erbas T, Sokmensuer C, Erbas B, Cila A. Amyloid goiter and hypopituitarism in a patient with systemic amyloidosis. Amyloid 18: 32-34, 2011.

33. el-Reshaid KA, Hakim AA, Hourani HA, Seshadri MS. Endocrine abnormalities in patients with amyloidosis. Ren Fail 16: 725-730, 1994.

34. Ramirez G, Gomez-Sanchez C, Meikle WA, Jubiz W. Evaluation of the hypothalamic hypophyseal adrenal axis in patients receiving long-term hemodialysis. Arch Intern Med 142: 1448-1452, 1982.

(C) 2014 The Japanese Society of Internal Medicine http://www.naika.or.jp/imonline/index.html 